Check for updates

Cite this: Mater. Chem. Front. 2018, 2, 1310

Received 1st December 2017, Accepted 16th March 2018

DOI: $10.1039 / \mathrm{c} 7 \mathrm{qm} 00551 \mathrm{~b}$

rsc.li/frontiers-materials

\section{Tetraphenylpyrazine-based luminogens with full-colour emission $\dagger$}

\author{
Lingxiang Pan, ${ }^{a}$ Yuanjing Cai, (D) ${ }^{a}$ Haozhong Wu, ${ }^{a}$ Fan Zhou, ${ }^{a}$ Anjun Qin, (D) *a \\ Zhiming Wang*a and Ben Zhong Tang*ab
}

\begin{abstract}
Aggregation-induced emission (AIE) has become a hot research topic. The generation of new AlE luminogens (AIEgens) will further promote the development of the AIE area. Currently, AIEgens with different emission colours have been reported. However, full-colour emissive luminogens based on a single AIE core and the structure-property relationship are rarely reported. To tackle this challenge, in this work, six tetraphenylpyrazine (TPP)-based luminogens DTPP, DTPP-Ph, DTPP-BT, DTPP-T, DTPP-TO and DTPP-TBTT are rationally designed by varying the effective conjugation and donor-acceptor units and facilely prepared under mild reaction conditions. The photophysical property investigation shows that they exhibit bright blue to red emission in the film states, covering the whole visible light range. Through systematic experimental investigations and theoretical calculations, their structure-photophysical property, and the competition of restriction of intramolecular motion and intramolecular charge-transfer are unambiguously elaborated. Moreover, DTPP-BT can be used as a reversible mechanochromic material and an indicator to show the exciton recombination area in OLEDs. This work provides guidance for further design of AlEgens with high efficiency and full-colour emission from a single AIE core.
\end{abstract}

\section{Introduction}

Recent years have witnessed the rapid development of organic light-emitting diodes (OLEDs) in full-colour display and white light emission. Efficient organic full-colour light-emitting materials are in great demand. ${ }^{1}$ However, most traditional organic fluorophores are highly emissive in dilute solutions, and their emission is weakened or totally quenched in the aggregate or solid state. In other words, these fluorophores suffer from the aggregation-caused quenching (ACQ) effect, which has become a thorny obstacle for their high-tech applications. ${ }^{2}$ To avoid the negative ACQ effect, chemical, physical and engineering approaches have been adopted. However, limited success has been achieved due to the natural process of molecular aggregation. ${ }^{3}$

Instead of fighting against the molecular aggregation in enhancing the emission of fluorophores, we found that molecular

\footnotetext{
${ }^{a}$ State Key Laboratory of Luminescent Materials and Devices, Center for Aggregation-Induced Emission, South China University of Technology, Guangzhou 510640, China. E-mail: msqinaj@scut.edu.cn,wangzhiming@scut.edu.cn ${ }^{b}$ Department of Chemistry, Hong Kong Branch of Chinese National Engineering Research Center for Tissue Restoration and Reconstruction, The Hong Kong University of Science \& Technology, Clear Water Bay, Kowloon, Hong Kong, China. E-mail: tangbenz@ust.hk

$\dagger$ Electronic supplementary information (ESI) available: ${ }^{1} \mathrm{H}$ and ${ }^{13} \mathrm{C}$ NMR spectra, HRMS spectra, photoluminescence spectra, the theoretical and experimental energy levels, time-resolved fluorescence spectra, performances of the electroluminescence devices. See DOI: 10.1039/c7qm00551b
}

aggregation can enhance the emission of luminogens in 2001 . Propeller-shaped molecules, such as siloles, are non-emissive in dilute solutions but remarkably emissive upon the formation of aggregates or fabrication into solid films. Accordingly, we termed this unique phenomenon as "aggregation-induced emission (AIE)". ${ }^{2 a-c, 4-12}$ Through systematic experimental investigations and theoretical simulation, restriction of intramolecular motion $(\mathrm{RIM}),{ }^{10}$ including restriction of intramolecular rotation (RIR) $)^{2 a, 11}$ and restriction of intramolecular vibration (RIV), ${ }^{12}$ has been rationalized as the mechanism of AIE. Under this guidance, many AIE cores, like silole, ${ }^{5}$ tetraphenylethylene (TPE), ${ }^{6}$ distyrylanthracene (DSA), ${ }^{7}$ tetraphenylbenzene, ${ }^{8 a}$ tetraphenylpyrrole, ${ }^{8 b}$ multiphenyl substituted 1,3-butadiene ${ }^{8 c}$ and others, ${ }^{9}$ have been prepared and applied in optoelectronics, sensing, and biological fields.

Recently, we made progress in developing another AIE core, namely tetraphenylpyrazine (TPP), which combines the advantages of TPE's facile preparation and silole's high thermal stability. ${ }^{13}$ Moreover, thanks to its easy functionalization, various substitutes have been attached to TPP and versatile properties have been realized. For example, the triphenylamine-modified TPP derivative shows high performance as an emitting layer in OLED application owing to its low electron affinity and good electron injection ability. ${ }^{14}$ By combining its aforementioned advantages, in this paper, we further developed TPP-based luminogens and widened their emission from the ultraviolet region at $390 \mathrm{~nm}$ to the whole visible light by tuning the conjugation and attaching the 

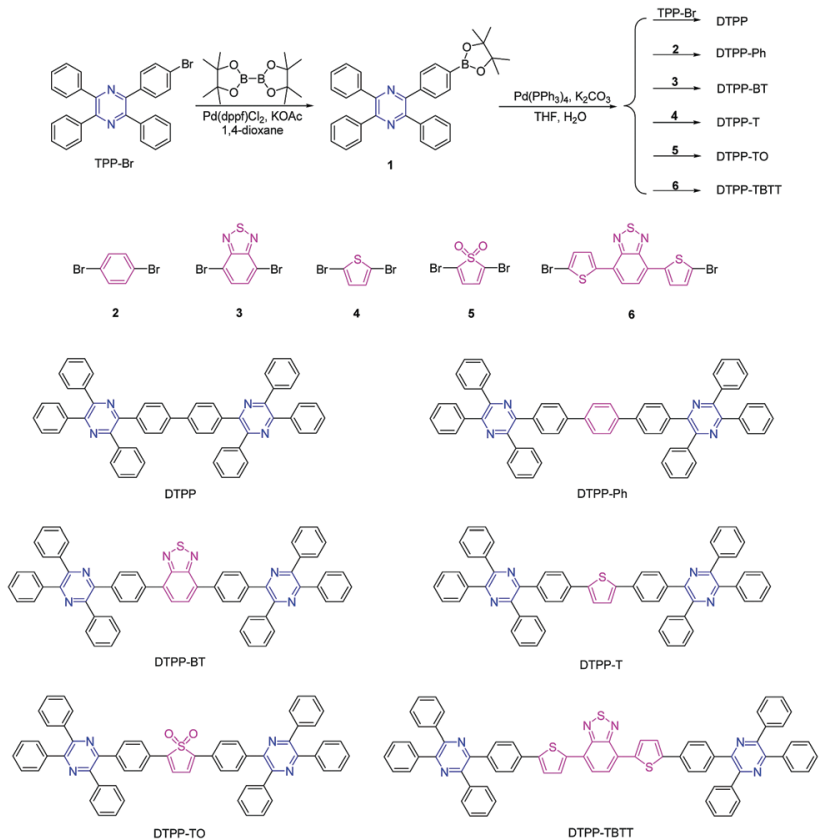

Scheme 1 Synthetic routes to TPP derivatives of DTPP, DTPP-Ph, DTPP-BT, DTPP-T, DTPP-TO and DTPP-TBTT.

electron-donating groups, such as thienyl and phenyl groups, and/or electron-accepting groups, such as benzothiadiazole (BT) and thienyl dioxide (TO), where the intramolecular charge transfer (ICT) will readily occur and in turn the emission is redshifted (Scheme 1). The strategy in this work provides guidance for designing highly efficient and full-colour emissive luminogens based on other AIE cores.

\section{Results and discussion}

\section{Synthesis}

The TPP derivatives of DTPP, DTPP-Ph, DTPP-BT, DTPP-T, DTPP-TO and DTPP-TBTT were facilely prepared according to the routes shown in Scheme 1 (the detailed synthetic procedures and characterization data are given in the ESI $\dagger$ ). The key intermediate of 1 was synthesized by the reaction of TPP-Br, ${ }^{14,15}$ and bis(pinacolato)diboron in a yield of $89.7 \%$. Then, 1 was subjected to react with TPP-Br or dibromo-substituted compounds of 2, 3, 4, 5 and 6 in the presence of the catalyst of $\mathrm{Pd}\left(\mathrm{PPh}_{3}\right)_{4}$, and DTPP, DTPP-Ph, DTPP-BT, DTPP-T, DTPP-TO and DTPP-TBTT were readily generated, respectively. These TPP derivatives were fully characterized by NMR spectroscopy and high resolution mass spectroscopy, and satisfactory results corresponding to their structures were obtained (Fig. S1-S20, ESI $\dagger$ ). Moreover, these compounds are soluble in commonly used organic solvents, such as dichloromethane, chloroform, and tetrahydrofuran (THF), but insoluble in water. They are also thermally stable. As shown in Fig. S21A (ESI $\dagger$ ), the temperatures of $5 \%$ weight loss of DTPP, DTPP-Ph, DTPP-BT, DTPP-T and TPP-TBTT are as high as 402, 442, 496, 476 and $526^{\circ} \mathrm{C}$, respectively, whereas TPP-TO begins to lose its weight at a temperature of $240{ }^{\circ} \mathrm{C}$ probably due to the decomposition of thiophene dioxide. Moreover, their glass transition temperatures $\left(T_{\mathrm{g}}\right)$ were also measured (Fig. S21B, ESI $\dagger$ ). We cannot observe the $T_{\mathrm{g}}$ of DTPP and DTPP-TO during the testing process, whereas those of the others were recorded ranging from 146 to $175{ }^{\circ} \mathrm{C}$, implying that they exhibit enough morphological stability in OLED application.

\section{Photophysical properties}

According to our design strategy, these luminogens should emit light covering the whole visible wavelength range. Indeed, the powders of these TPP derivatives emit blue to red light upon irradiation with a UV lamp (Fig. 1). Since this is the first time to realize the full-colour emission of TPP-based luminogens, we systematically investigate their photophysical properties.

Fig. 2A shows the absorption spectra of these TPP-based luminogens in THF. DTPP, DTPP-Ph and DTPP-T have similar absorption profiles with peaks at 353, 354 and $385 \mathrm{~nm}$, respectively, while those of DTPP-BT, DTPP-TO and DTPP-TBTT exhibit two absorption peaks with longer wavelengths at 403, 426 and $508 \mathrm{~nm}$, respectively. This difference could be ascribed to ICT process. Although TPP has been proven to be an electron-withdrawing group in our previous work, ${ }^{14 a}$ no peaks associated with ICT were found when the phenyl or thienyl group was inserted between two TPP units, which is probably due to the structural symmetry. However, when strong electron-accepting groups, such as BT, TO and thienyl-BT-thienyl, are inserted, the ICT process readily occurred, in which the TPP unit might serve as an electron-donating group. These results suggest that TPP might be potentially used as a bipolar moiety.

Next, the photoluminescence (PL) spectra of these luminogens were measured to further distinguish their difference in the ICT process. The results show that these luminogens emit in the range of 430-612 nm in THF (Table 1, Fig. 2B and Fig. S22, ESI $\dagger$ ).

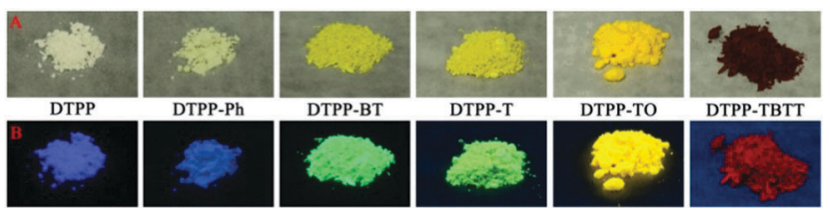

Fig. 1 Photos of DTPP, DTPP-Ph, DTPP-BT, DTPP-T, DTPP-TO and DTPP-TBTT in amorphous powders, taken under the illumination of (A) a daylight lamp and (B) an UV lamp (365 nm).
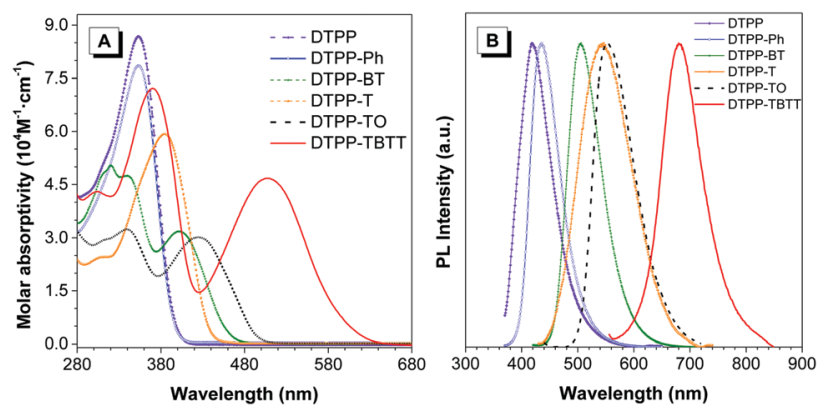

Fig. 2 (A) Absorption of THF solutions $(10 \mu \mathrm{M})$ and (B) photoluminescence spectra of films of DTPP, DTPP-Ph, DTPP-BT, DTPP-T, DTPP-TO and DTPP-TBTT. 
Table 1 The photophysical properties of DTPP, DTPP-Ph, DTPP-BT, DTPP-T, DTPP-TO and DTPP-TBTT

\begin{tabular}{|c|c|c|c|c|c|c|c|c|c|c|c|}
\hline \multirow[b]{2}{*}{ Compound } & \multirow[b]{2}{*}{$\lambda_{\mathrm{abs}}{ }^{a}$} & \multicolumn{2}{|c|}{$\underline{\lambda_{\mathrm{em}}{ }^{b} / \mathrm{nm}}$} & \multicolumn{2}{|c|}{$\underline{\Phi_{\mathrm{F}}{ }^{c} / \%}$} & \multicolumn{2}{|l|}{$\tau^{d} / \mathrm{ns}$} & \multicolumn{2}{|c|}{$\underline{K_{\mathrm{r}}^{e}\left(10^{7} \mathrm{~s}^{-1}\right)}$} & \multicolumn{2}{|c|}{$\underline{K_{\mathrm{nr}}^{f}}\left(10^{7} \mathrm{~s}^{-1}\right)$} \\
\hline & & Soln & Film & Soln & Film & Soln & Film & Soln & Film & Soln & Film \\
\hline DTPP & 353 & 430 & 418 & 0.9 & 16.9 & 0.41 & 0.53 & 2.2 & 31.9 & 241.7 & 156.8 \\
\hline DTPP-Ph & 354 & 434 & 436 & 1.0 & 28.3 & 0.41 & 0.55 & 2.4 & 51.5 & 241.5 & 130.4 \\
\hline DTPP-BT & 340,403 & 509 & 506 & 79.5 & 62.1 & 3.19 & 1.81 & 24.9 & 34.3 & 6.4 & 20.9 \\
\hline DTPP-T & 385 & 455 & 546 & 36.5 & 21.7 & 0.67 & 0.84 & 54.5 & 25.8 & 94.8 & 93.2 \\
\hline DTPP-TO & 341,426 & 529 & 552 & 55.2 & 33.3 & 1.98 & 1.47 & 27.9 & 22.7 & 22.6 & 45.4 \\
\hline DTPP-TBTT & 370,508 & 612 & 680 & 66.1 & 13.0 & 5.26 & 3.66 & 12.6 & 3.6 & 6.4 & 23.8 \\
\hline
\end{tabular}

${ }^{a}$ Maximum absorption wavelength, concentration: $10 \mu \mathrm{M} .{ }^{b}$ Maximum emission wavelength, soln: THF solution, film: droplet film. ${ }^{c}$ Absolute fluorescence quantum efficiency. ${ }^{d}$ Fluorescence lifetime. ${ }^{e} K_{\mathrm{r}}=\Phi_{\mathrm{F}} / \tau .{ }^{f} K_{\mathrm{nr}}=\left(1-\Phi_{\mathrm{F}}\right) / \tau$.

Interestingly, the emission of DTPP-T, DTPP-TO and DTPP-TBTT in solutions is remarkably red-shifted compared to those in films, whereas little change was found in the others.

TPP is a good AIEgen. Are TPP-based luminogens also AIE active? To answer this question, we investigated their emission behaviours in THF/water mixtures with different water fractions $\left(f_{\mathrm{w}}\right)$ (Fig. 3 and Fig. S23, ESI $\dagger$ ). The results show that DTPP and DTPP-Ph are weakly emissive in THF but their emission is greatly intensified in THF/water mixtures with $f_{\mathrm{w}}$ of more than $50 \%$, exhibiting typical aggregation-enhanced emission (AEE) characteristics. Interestingly, as shown in Fig. $3 \mathrm{~A}$, with $f_{\mathrm{w}}$ from 0 to $80 \%$, the emission peaks of DTPP in THF/water mixtures are blue-shifted from 430 to $421 \mathrm{~nm}$. This phenomenon could be attributed to the formation of nanocrystals as generally observed in TPE-based systems, ${ }^{16}$ while the others exhibit a typical ACQ effect. The absolute quantum yields $\left(\Phi_{\mathrm{F}}\right)$ also confirm the PL measurements. As shown in Table 1, AEE-active DTPP and
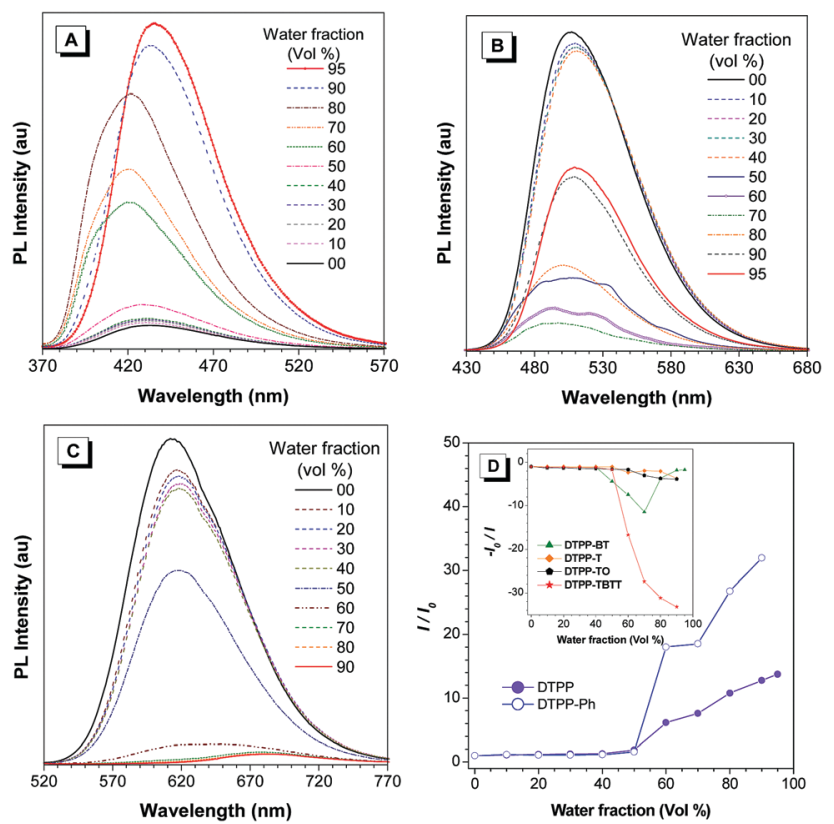

Fig. 3 PL spectra of (A) DTPP, (B) DTPP-BT, and (C) DTPP-TBTT in THF/ water mixtures with different water fractions; $\lambda_{\text {ex }}: 353,403$ and $508 \mathrm{~nm}$ for DTPP, DTPP-BT and DTPP-TBTT, respectively; concentration: $10 \mu \mathrm{M}$. (D) and inset: plot of relative intensity of TPP-based luminogens versus water fraction in THF/water mixtures. $I_{0}$ refers to the original emission intensity in THF.
DTPP-Ph possess quite low $\Phi_{\mathrm{F}}(\sim 1 \%)$ in THF solutions; however, the values greatly increase to 16.9 and $28.3 \%$ in their film states, respectively. The others show high $\Phi_{\mathrm{F}}$ in solutions, but the values decrease upon fabrication into thin films.

These results indicate that for DTPP and DTPP-Ph, the RIM process plays a crucial role in intensifying the emission in the aggregate and film states, whereas for DTPP-BT, DTPP-TO, and DTPP-TBTT, both RIM and ICT processes affect their emission behaviours, and the latter is much stronger than the former. In addition, the ACQ effect of DTPP-T that contains no strong electron-accepting but donating thienyl group might be probably due to the planarization of the structure, which is proved by the red-shifted emission in solution compared to that in the film state.

In order to further understand the photophysical properties of these TPP-based luminogens, their time-resolved fluorescence was measured in THF solution and film states (Fig. S24, ESI $\dagger$ ), and the radiative and non-radiative decay rates were calculated and are listed in Table 1. Generally, the RIM process would lead to the decrease of non-radiative decay rate $\left(K_{\mathrm{nr}}\right)$. Thus, this parameter is used to check the occurrence of the RIM process.

Compared with the transition rates in solution, the radiative decay rates $\left(K_{\mathrm{r}}\right)$ of DTPP and DTPP-Ph in the film state become much larger. At the same time, the values of $K_{\mathrm{nr}}$ decrease. These data imply that the improvement in the fluorescence efficiency of DTPP and DTPP-Ph could be ascribed to the RIM process. For DTPP-TO and DTPP-TBTT, the $K_{\mathrm{r}}$ values become smaller, while the $K_{\mathrm{nr}}$ values increase, indicating that the RIM effect on the improved efficiency is far less than that of the ICT process. As a result, they suffer from the ACQ effect. In addition, as a special example, both $K_{\mathrm{r}}$ and $K_{\mathrm{nr}}$ values of DTPP-BT in the film state become larger simultaneously than those in the solution state, suggesting that the RIM and ICT processes are all active, and the effect of the latter is a little stronger than that of the former. Moreover, for DTPP-T, both $K_{\mathrm{r}}$ and $K_{\mathrm{nr}}$ values decrease from the solution to film state, and the decrease of $K_{\mathrm{r}}$ is much faster than that of $K_{\mathrm{nr}}$, which leads to the quenching of its fluorescence.

\section{Theoretical calculations}

We also studied the intrinsic photophysical processes of these TPP-based luminogens using density function theory (DFT) of B3LYP with the 6-31G(d) basis set. As shown in Fig. 4 and Table 2, the dihedral angles of periphery phenyl rings 


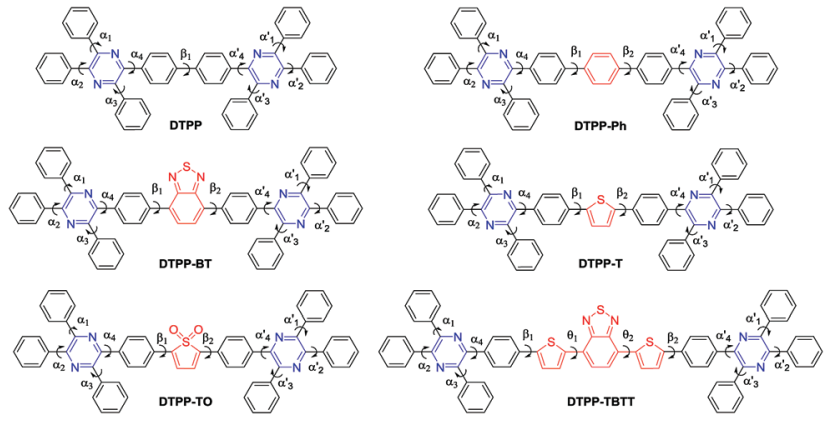

Fig. 4 The marks of the twisting angles in the optimized molecular structures of DTPP, DTPP-Ph, DTPP-BT, DTPP-T, DTPP-TO and DTPP-TBTT.

connected with the pyrazine cores are around $36^{\circ}$ in the optimized TPP-based luminogens. This structural configuration is beneficial for obstructing the intermolecular $\pi-\pi$ stacking and improving fluorescence efficiency in the aggregate state via the RIM process.

However, the distribution of HOMOs and LUMOs in their molecular structures is quite different (Fig. 5). For DTPP and DTPP-Ph, their HOMOs and LUMOs are almost located on the whole molecules, suggesting that the RIM process is a potential and key factor in influencing radiation transition behaviors. Meanwhile, the ICT process is negligible for DTPP and DTPP-Ph. Thus, we can draw a conclusion that the AEE feature of these two luminogens is due to the RIM process.

For DTPP-TO and DTPP-TBTT, obvious ICT processes take place and a small amount of electron clouds are located on the periphery phenyl rings. These results suggest that the PL is dominated by the ICT process and RIM plays a less important role. Hence, the $\Phi_{\mathrm{F}}$ values of DTPP-TO and DTPP-TBTT decrease from the solution to film state. Similarly, a small amount of electron clouds are located on the periphery phenyl rings of DTPP-T as shown in Fig. 5. As a result, no obvious ICT process is observed. However, the planar central structure of phenyl-thienylphenyl moieties might facilitate the intermolecular interactions in the aggregate state, which leads to the decrease in its emission. This conclusion could well explain its aforementioned absorption and emission behaviours.

Meanwhile, for DTPP-BT, the distribution of the HOMO and LUMO implies the presence of the ICT process like DTPP-TBTT. In addition, the electron clouds in the HOMO are also partially located on the periphery phenyl rings like DTPP. These results suggest that both RIM and ICT processes influence its emission in the aggregate state. The balance of these two competitive processes makes DTPP-BT highly emissive both in solution and film states. ${ }^{17}$

\section{Mechanochromic properties}

Among the prepared TPP-based luminogens, DTPP-BT displays fluorescent mechanochromic behaviour. As shown in Fig. 7, the as-prepared green emissive powder peaked at $502 \mathrm{~nm}$ could be converted to a yellow emissive one with peak shifted to $518 \mathrm{~nm}$ upon grinding using a pestle. Thus, the characters of "AIE" could be clearly seen if written by the pestle. When fuming the yellow emissive powder with $\mathrm{CH}_{2} \mathrm{Cl}_{2}$, it readily recovered to the green emission. Moreover, this change is reversible without distinct waveform variations of the two states (Fig. 8A). The powder X-ray diffraction (PXRD) technique was used to reveal the reason for the mechanochromic behaviour of DTPP-BT, and the results indicate that this phenomenon occurs due to the change in molecular packing modes. ${ }^{6 b}$ As shown in Fig. 8B, the diffraction patterns of the as-prepared and the solvent fumed powders clearly exhibit sharp and intense reflections, indicating DTPP-BT form microcrystals. In contrast, the ground powders do not exhibit any noticeable diffraction in the PXRD profile, reflecting its amorphous feature.

\section{Electrochemical properties}

Cyclic voltammetry (CV) measurements were carried out to evaluate the practical energy levels of these TPP-based luminogens. The onset potentials of DTPP, DTPP-Ph, DTPP-BT, DTPP-T, DTPP-TO and DTPP-TBTT were deduced to be 1.34, 1.41, 1.38, 1.15, 1.45 and $0.93 \mathrm{eV}$, respectively (Fig. 6). The HOMO energy levels are estimated from the onset oxidation potentials according to the equation: $\mathrm{HOMO}=-\left(4.4+E_{\text {onset }}\right) \mathrm{eV}$, and the LUMO energy levels (LUMO $=\mathrm{HOMO}+E_{\mathrm{g}}$ ) are determined by the optical band gaps $\left(E_{\mathrm{g}}=1240 / \lambda_{\text {onset }}\right.$, where the $\lambda_{\text {onset }}$ is calculated by onset absorption). ${ }^{6 d, 16,17}$ The HOMO energy levels were thus calculated to be $-5.74,-5.81,-5.78,-5.55,-5.85$ and $-5.33 \mathrm{eV}$, and the LUMO energy levels are $-2.61,-2.68,-3.08,-2.73,-3.35$ and $-3.26 \mathrm{eV}$ for DTPP, DTPP-Ph, DTPP-BT, DTPP-T, DTPP-TO and DTPP-TBTT, respectively (Table S1, ESI $\dagger$ ). The LUMO energy levels of these TPP-based luminogens are close to or even lower than those of the widely used electron-transporting materials, such as $\mathrm{Alq}_{3}(-3.0 \mathrm{eV})^{18}$ and TPBi $(-2.7 \mathrm{eV}){ }^{19}$ suggesting that they are promising candidates for OLED application.

Table 2 The twisting angles in DTPP, DTPP-Ph, DTPP-BT, DTPP-T, DTPP-TO and DTPP-TBTT

Twisting angle ${ }^{a}\left({ }^{\circ}\right)$

\begin{tabular}{|c|c|c|c|c|c|c|c|c|c|c|c|c|}
\hline \multirow[b]{2}{*}{ Luminogens } & \\
\hline & $\alpha_{1}$ & $\alpha_{2}$ & $\alpha_{3}$ & $\alpha_{4}$ & $\alpha_{1}^{\prime}$ & $\alpha_{2}^{\prime}$ & $\alpha_{3}^{\prime}$ & $\alpha_{4}^{\prime}$ & $\beta_{1}$ & $\beta_{2}$ & $\theta_{1}$ & $\theta_{2}$ \\
\hline DTPP & 36.00 & 35.62 & 36.26 & 34.78 & 35.62 & 36.00 & 36.26 & 34.78 & 35.47 & & & \\
\hline DTPP-Ph & 35.95 & 35.84 & 36.05 & 35.11 & 35.92 & 35.83 & 36.29 & 34.62 & 35.93 & 35.26 & & \\
\hline DTPP-BT & 36.04 & 35.53 & 36.28 & 34.80 & 35.88 & 35.50 & 35.91 & 35.11 & 34.74 & 34.61 & & \\
\hline DTPP-T & 36.30 & 35.90 & 36.20 & 34.53 & 33.74 & 36.29 & 36.20 & 34.53 & 20.86 & 20.86 & & \\
\hline DTPP-TO & 35.81 & 35.27 & 35.85 & 34.46 & 35.94 & 35.30 & 36.47 & 34.56 & 22.64 & 19.97 & & \\
\hline DTPP-TBTT & 36.38 & 35.57 & 36.46 & 33.75 & 36.26 & 35.30 & 36.36 & 33.55 & 21.09 & 23.50 & 4.90 & 7.58 \\
\hline
\end{tabular}

${ }^{a}$ The marks of the twist angles are shown in Fig. 4 . 

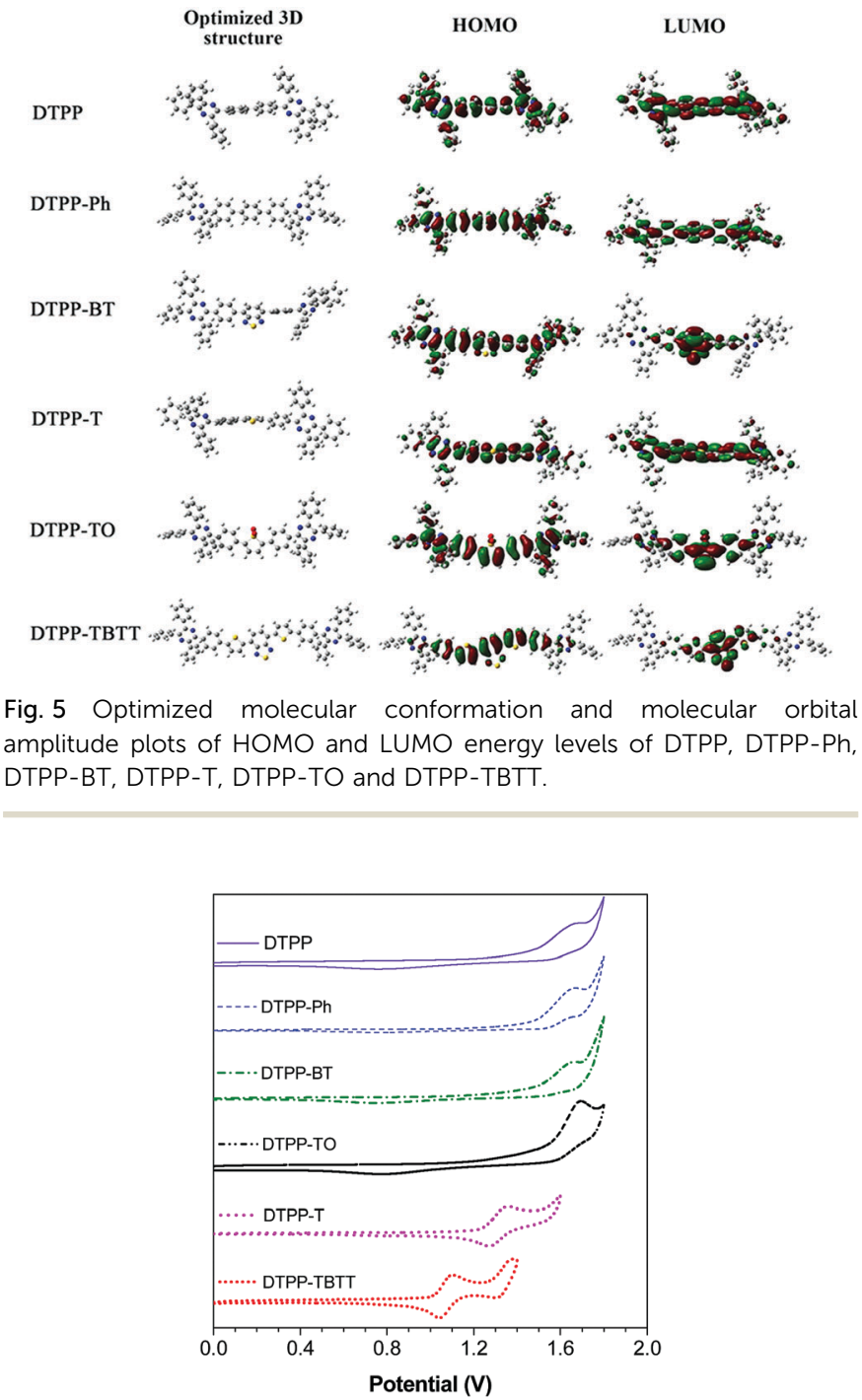

Fig. 6 Cyclic voltammograms of DTPP, DTPP-Ph, DTPP-BT, DTPP-T, DTPP-TO and DTPP-TBTT, measured in dichloromethane containing $0.1 \mathrm{M}$ tetra- $n$-butylammonium hexafluorophosphate, scan rate: $100 \mathrm{mV} \mathrm{s}^{-1}$.

\section{Electroluminescence (EL)}

Thanks to the balance between the RIM and ICT processes of DTPP-BT, it shows a high $\Phi_{\mathrm{F}}$ of $62.1 \%$ in the film state. We thus used it as an emitting layer to fabricate the non-doped OLED with the device configuration of ITO/NPB (60 nm)/DTPP-BT $(20 \mathrm{~nm}) / \mathrm{TPBi}(40 \mathrm{~nm}) / \mathrm{LiF}(1 \mathrm{~nm}) / \mathrm{Al}$ (device I), where $N, N$-bis(1naphthyl)- $N, N$-diphenylbenzidine (NPB) serves as the hole-transporting layer, 2,2' $2^{\prime \prime}$-(1,3,5-benzinetriyl)tris-(1-phenyl-1- $H$-benzimid-
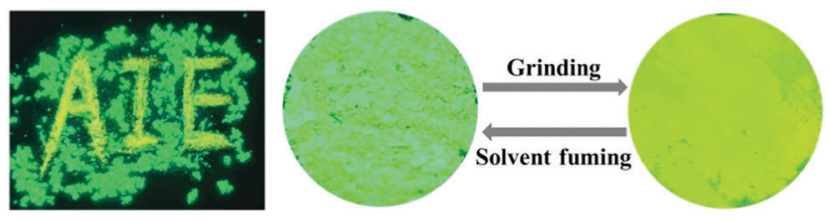

Fig. 7 Photographs of DTPP-BT in the pristine state and its ground or solvent fuming samples under a UV lamp.
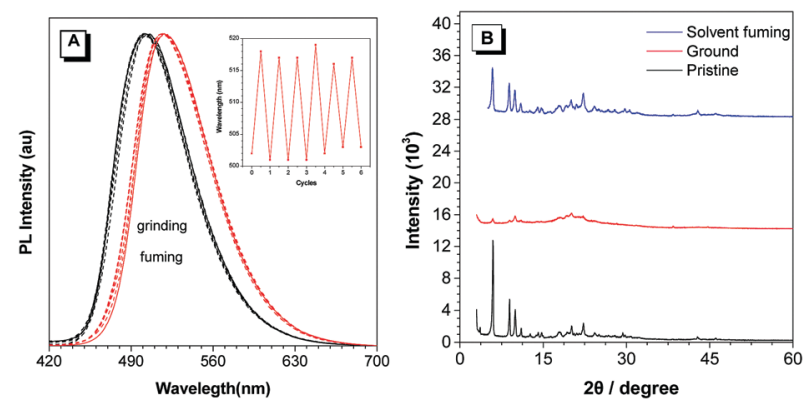

Fig. 8 (A) Normalized PL curves of DTPP-BT with reversible grinding and fuming. The inset shows the emission wavelength of the repeated grinding and fuming treatments of DTPP-BT. (B) XRD profiles of DTPP-BT in different solid phases.
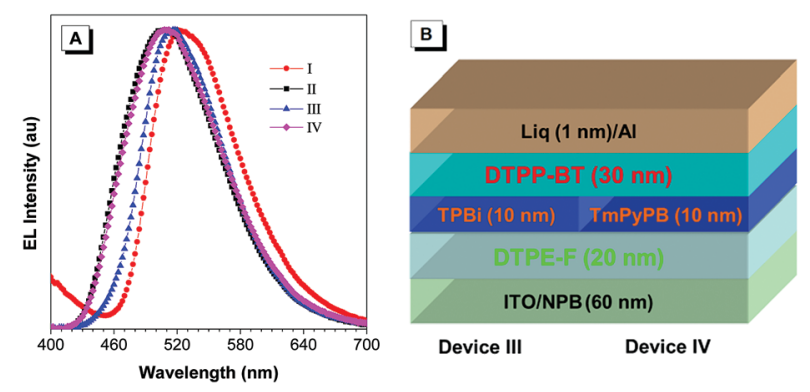

Fig. 9 (A) EL spectra of devices I-IV, and (B) the configuration diagrams of devices III and IV.

azole) (TPBi) works as the electron-transporting layer, and LiF functions as the electron-injecting layer. ${ }^{20}$ As shown in Fig. 9 and Table 3 (for details, please refer to Fig. S25, ESI $\dagger$ ), device I exhibits green EL at $520 \mathrm{~nm}$ with Commission International d'Eclairage (CIE) chromaticity coordinates of $(0.319,0.543)$. The maximum luminance $\left(L_{\mathrm{max}}\right)$, power $\left(\eta_{\mathrm{P}, \max }\right)$, current $\left(\eta_{\mathrm{C}, \mathrm{max}}\right)$, and external quantum $\left(\eta_{\text {ext,max }}\right)$ efficiencies of device I were measured to be $3173 \mathrm{~cd} \mathrm{~m}^{-2}, 0.77 \mathrm{~lm} \mathrm{~W}^{-1}, 1.25 \mathrm{~cd} \mathrm{~A}^{-1}$, and $0.4 \%$, respectively. To explain the relatively low performance of device I, a more precise LUMO value of DTPP-BT was estimated from the reduction potential in the $\mathrm{CV}$ curve (Fig. S26, ESI $\dagger$ ), and this value was deduced to be $-3.38 \mathrm{eV}$, which is lower than the one determined from the optical band gap $(-3.08 \mathrm{eV})$. By comparison, DTPP-TBTT and DTPP-TO were measured under the same conditions, and the values were calculated at $-3.39 \mathrm{eV}$ and -3.50 (Fig. S26, ESI $\dagger$ ). The difference between optical band gaps and electro band gaps of these luminogens might originate from their stronger donor-acceptor conjugation effect. This explanation is further confirmed by the red-shifted EL emission of DTPP-BT from its film $(506 \mathrm{~nm})$ to device $(520 \mathrm{~nm})$. The more accurate LUMO value also provides a more reasonable explanation for the lower OLED performance.

Meanwhile, the low LUMO energy level of DTPP-BT makes it potentially useful as electron-modified layer. To confirm this hypothesis, we adopted tetraphenylethylene-furan (TPE-F), our previously reported AIEgen from which excellent device performance was achieved (device II in this paper), ${ }^{21}$ as an emitting layer, DTPP-BT as an electron-injecting/transporting layer, and 
Table 3 EL performances of non-doped OLEDs based on DTPP-BT and DTPP-T

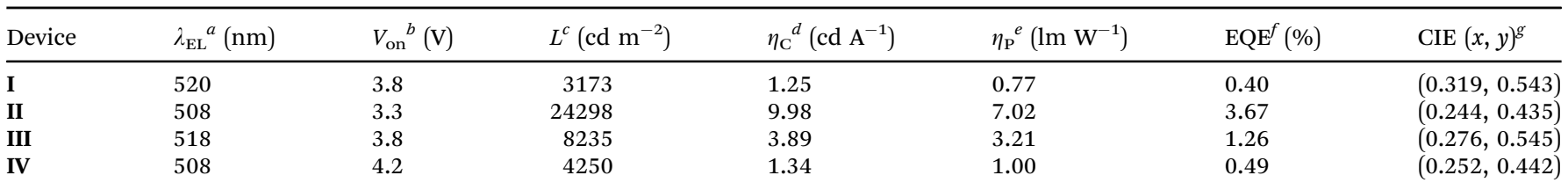

${ }^{a} \lambda_{\mathrm{EL}}=$ EL maximum. ${ }^{b} V_{\mathrm{on}}=$ turn-on voltage at $1 \mathrm{~cd} \mathrm{~m}^{-2} \cdot{ }^{c} L_{\max }=$ maximum luminance. ${ }^{d} \eta_{\mathrm{c}, \max }=$ maximum current efficiency. ${ }^{e} \eta_{\mathrm{P}, \text { max }}=$ maximum power efficiency. ${ }^{f} \eta_{\text {ext,max }}=$ maximum external quantum efficiency. ${ }^{g}$ CIE $=$ Commission International de I'Eclairage coordinates.

TPBi or 1,3,5-tri[(3-pyridyl)-phen-3-yl]benzene (TmPyPB) as a hole-blocking layer to fabricate devices (III and IV). Device III (configuration: ITO/NPB (60 nm)/DTPE-F (20 nm)/TPBi $(10 \mathrm{~nm}) /$ DTPP-BT $(30 \mathrm{~nm}) / \mathrm{LiF}(1 \mathrm{~nm}) / \mathrm{Al})$ and device IV (configuration: ITO/NPB (60 nm)/DTPE-F (20 nm)/TmPyPB(10 nm)/DTPP-BT $(30 \mathrm{~nm}) / \mathrm{LiF}(1 \mathrm{~nm}) / \mathrm{Al})$ exhibit good performance with bright green emission as shown in Table 3. However, the areas of exciton recombination in devices III and IV are different as indicated by their EL peaks. In device III, the EL is peaked at around $518 \mathrm{~nm}$, which is similar to that of device I, implying that the exciton recombination area might be in the DTPP-BT layer instead of the emitting layer. In device IV, the EL emission is peaked at $508 \mathrm{~nm}$, which is similar with that of device II, suggesting that the exciton recombination area is in the emitting layer.

Since the difference of devices III and IV is mainly in the hole-blocking layer of TPBi and TmPyPB, these results indicate that DTPP-BT could be used as an indicator to show the exciton recombination area and estimate the hole blocking capability of some electron-modified materials.

\section{Conclusion}

In this paper, six TPP-based luminogens DTPP, DTPP-Ph, DTPP-BT, DTPP-T, DTPP-TO, DTPP-TBTT were rationally designed via tuning their conjugation and donor-acceptor units and facilely prepared under mild reaction conditions. They exhibit blue, green, yellow and red emission in film states, almost covering the entire visible range upon UV irradiation. This is the first time to realize full-colour emission based on the TPP core. Through systematic experimental investigations and theoretical calculations, the structure-photophysical property and the competition of RIM and ICT of these luminogens have been unambiguously revealed. Luminogens without ICT processes exhibit AEE features, whereas compounds with ICT process show an ACQ effect, and the balance of the RIM and ICT processes makes DTPP-BT highly emissive both in the solution and film states. Moreover, DTPP-BT possesses reversible mechanochromic effect and can be used as an indicator to show the exciton recombination area in OLEDs. Thus, this work not only further develops the TPP-based AIEgens but also provides guidance for the construction of highly efficient and full-colour emissive AIEgens with other AIE-cores.

\section{Conflicts of interest}

There are no conflicts to declare.

\section{Acknowledgements}

This work was financially supported by the National Natural Science Foundation of China (21788102, 21525417, 51703066 and 51673118), the National Program for Support of Top-Notch Young Professionals, the Natural Science Foundation of Guangdong Province (2016A030312002), the Fundamental Research Funds for the Central Universities (2015ZY013 and 2017MS015) and the Innovation and Technology Commission of Hong Kong (ITC-CNERC14S01). Y. J. C. acknowledges the support from the China Postdoctoral Science Foundation (2016 M602463). A. J. Q. and B. Z. T. acknowledge the support from the Guangdong Innovative Research Team Program (201101C0105067115).

\section{Notes and references}

1 (a) C. W. Tang and S. A. VanSlyke, Appl. Phys. Lett., 1987, 51, 913; (b) H. Uoyama, K. Goushi, K. Shizu, H. Nomura and C. Adachi, Nature, 2012, 492, 234; (c) K. Hitoshi and H. Chishio, Sci. Technol. Adv. Mater., 2014, 15, 034201; (d) S. Kim, B. Kim, J. Lee, H. Shin, Y.-I. Park and J. Park, Mater. Sci. Eng., R, 2016, 99, 1; (e) C. Wang and Z. Li, Mater. Chem. Front., 2017, 1, 2174; $(f)$ Z. Li, Sci. China: Chem., 2017, 8, 1107; ( $g$ ) J. Yang, L. Li, Y. Yu, Z. Ren, Q. Peng, S. Ye, Q. Li and Z. Li, Mater. Chem. Front., 2017, 1, 91.

2 (a) Y. Hong, J. W. Y. Lam and B. Z. Tang, Chem. Commun., 2009, 4332; (b) Z. Zhao, P. Lu, J. W. Y. Lam, Z. Wang, C. Y. K. Chan, H. H. Y. Sung, I. D. Williams, Y. Ma and B. Z. Tang, Chem. Sci., 2011, 2, 672; (c) J. Mei, N. L. C. Leung, R. T. K. Kwok, J. W. Y. Lam and B. Z. Tang, Chem. Rev., 2015, 115, 11718; (d) K.-Y. Pu and B. Liu, Adv. Funct. Mater., 2009, 19, 277; (e) M. Yamaguchi, S. Ito, A. Hirose, K. Tanaka and Y. Chujo, Mater. Chem. Front., 2017, 1, 1573.

3 (a) C.-T. Chen, Chem. Mater., 2004, 16, 4389; (b) S. Setayesh, A. C. Grimsdale, T. Weil, V. Enkelmann, K. Müllen, F. Meghdadi, E. J. W. List and G. Leising, J. Am. Chem. Soc., 2001, 123, 946.

4 J. Luo, Z. Xie, J. W. Y. Lam, L. Cheng, H. Chen, C. Qiu, H. S. Kwok, X. Zhan, Y. Liu, D. Zhu and B. Z. Tang, Chem. Commun., 2001, 1740.

5 (a) Y. Dong, J. W. Y. Lam, A. Qin, Z. Li, J. Liu, J. Sun, Y. Dong and B. Z. Tang, Chem. Phys. Lett., 2007, 446, 12; (b) H. Shi, J. Liu, J. Geng, B. Z. Tang and B. Liu, J. Am. Chem. Soc., 2012, 134, 9569; (c) Z. Zhao, Z. Wang, P. Lu, C. Y. K. Chan, D. Liu, J. W. Y. Lam, H. H. Y. Sung, I. D. Williams, Y. Ma and B. Z. Tang, Angew. Chem., 2009, 121, 7744; (d) B. Chen, 
G. Feng, B. He, C. Goh, S. Xu, G. Ramos-Ortiz, L. Aparicio-Ixta, J. Zhou, L. Ng, Z. Zhao, B. Liu and B. Z. Tang, Small, 2016, 12,782 .

6 (a) Y. Q. Dong, J. W. Y. Lam, A. J. Qin, J. Z. Liu, Z. Li and B. Z. Tang, Appl. Phys. Lett., 2007, 91, 011111; (b) Z. Zhao, J. W. Y. Lam and B. Z. Tang, J. Mater. Chem., 2012, 22, 23726; (c) G. Lin, H. Peng, L. Chen, H. Nie, W. Luo, Y. Li, S. Chen, R. Hu, A. Qin, Z. Zhao and B. Z. Tang, ACS Appl. Mater. Interfaces, 2016, 8, 16799; (d) F. Hu, G. X. Zhang, C. Zhan, W. Zhang, Y. L. Yan, Y. S. Zhao, H. B. Fu and D. Q. Zhang, Small, 2015, 11, 1335; (e) C. Zhan, X. You, G. X. Zhang and D. Q. Zhang, Chem. Rec., 2016, 4, 2142-2160; $(f)$ L. Wang, H. K. Zhang, A. Qin, Q. Jin, B. Z. Tang and J. Ji, Sci. China: Chem., 2016, 12, 1609.

7 (a) J. He, B. Xu, F. Chen, H. Xia, K. Li, L. Ye and W. Tian, J. Phys. Chem. C, 2009, 113, 9892; (b) X. Zhang, Z. Chi, B. Xu, L. Jiang, X. Zhou, Y. Zhang, S. Liu and J. Xu, Chem. Commun., 2012, 48, 10895; (c) H. Li, X. Zhang, Z. Chi, B. Xu, W. Zhou, S. Liu, Y. Zhang and J. Xu, Org. Lett., 2011, 13, 556.

8 (a) L. Z. Li, H. Nie, M. Chen, J. Z. Sun, A. J. Qin and B. Z. Tang, Faraday Discuss., 2017, 196, 245; (b) X. Feng, B. Tong, J. B. Shen, J. B. Shi, T. Y. Han, L. Chen, J. G. Zhi, P. Lu, Y. G. Ma and Y. P. Dong, J. Phys. Chem. B, 2010, 50, 16731; (c) F. Bu, E. Wang, Q. Peng, R. Hu, A. Qin, Z. Zhao and B. Z. Tang, Chem. - Eur. J., 2015, 21, 4440; (d) J. Yang, Z. Ren, B. Chen, M. Fang, Z. Zhao, B. Z. Tang, Q. Peng and Z. Li, J. Mater. Chem. C, 2017, 5, 9242.

9 (a) T. Matsumoto, H. Takamine, K. Tanaka and Y. Chujo, Mater. Chem. Front., 2017, 1, 2368; (b) J. Guo, S. Hu, W. Luo, R. Hu, A. Qin, Z. Zhao and B. Z. Tang, Chem. Commun., 2017, 53, 1463; (c) W. Huang, L. Sun, Z. W. Zheng, J. H. Su and H. Tian, Chem. Commun., 2015, 21, 4462; (d) H. Nie, K. Hu, Y. J. Cai, Q. Peng, Z. J. Zhao, R. R. Hu, J. W. Chen, S.-J. Su, A. Qin and B. Z. Tang, Mater. Chem. Front., 2017, 1, 1125; (e) Z. T. Wang, Y. Fang, J. Z. Sun, A. Qin and B. Z. Tang, Sci. China: Chem., 2013, 9, 1187-1190; $(f)$ J. Yang, Z. Ren, Z. Xie, Y. Liu, C. Wang, Y. Xie, Q. Peng, B. Xu, W. Tian,
F. Zhang, Z. Chi, Q. Li and Z. Li, Angew. Chem. Int. Ed., 2017, $56,880$.

10 J. Mei, Y. Hong, J. W. Y. Lam, A. Qin, Y. Tang and B. Z. Tang, Adv. Mater., 2014, 26, 5429.

11 Y. Hong, J. W. Y. Lam and B. Z. Tang, Chem. Soc. Rev., 2011, 40, 5361.

12 N. L. C. Leung, N. Xie, W. Yuan, Y. Liu, Q. Wu, Q. Peng, Q. Miao, J. W. Y. Lam and B. Z. Tang, Chem. - Eur. J., 2014, 20, 15349.

13 M. Chen, L. Li, H. Nie, J. Tong, L. Yan, B. Xu, J. Z. Sun, W. Tian, Z. Zhao, A. Qin and B. Z. Tang, Chem. Sci., 2015, 6, 1932.

14 (a) M. Chen, H. Nie, B. Song, L. Li, J. Z. Sun, A. Qin and B. Z. Tang, J. Mater. Chem. C, 2016, 4, 2901; (b) L. X. Pan, M. Chen, J. Liu, L. Xu, R. Hu, Z. Zhao, A. Qin and B. Z. Tang, Chin. J. Org. Chem., 2016, 36, 1316.

15 P. Karastatiris, J. A. Mikroyannidis, I. K. Spiliopoulos, A. P. Kulkarni and S. A. Jenekhe, Macromolecules, 2004, 37, 7867.

16 C. Y. K. Chan, Z. Zhao, J. W. Y. Lam, J. Liu, S. Chen, P. Lu, F. Mahtab, X. Chen, H. H. Y. Sung, H. S. Kwok, Y. Ma, I. D. Williams, K. S. Wong and B. Z. Tang, Adv. Funct. Mater., 2012, 22, 378.

17 L. Chen, G. Lin, H. Peng, H. Nie, Z. Zhuang, P. Shen, S. Ding, D. Huang, R. Hu, S. Chen, F. Huang, A. Qin, Z. Zhao and B. Z. Tang, J. Mater. Chem. C, 2016, 4, 5241.

18 S. Chen., Z. J. Zhao, B. Z. Tang and H. S. Kowk, J. Phys. D: Appl. Phys., 2010, 43, 095101.

19 Z. Gao, C. S. Lee, I. Bello, S. T. Lee, R.-M. Chen, T.-Y. Luh, J. Shi and C. W. Tang, Appl. Phys. Lett., 1999, 74, 865.

20 (a) Z. Chi, X. Zhang, B. Xu, X. Zhou, C. Ma, Y. Zhang, S. Liu and J. Xu, Chem. Soc. Rev., 2012, 41, 3878; (b) T. Jadhav, B. Dhokale, Y. Patil, S. M. Mobin and R. Misra, J. Phys. Chem. C, 2016, 120, 24030; (c) R. Rao, M. C.-W. Liao, W.-L. Su and S.-S. Sun, J. Mater. Chem. C, 2013, 1, 5491; (d) C. Dou, D. Chen, J. Iqbal, Y. Yuan, H. Zhang and Y. Wang, Langmuir, 2011, 27, 6323; (e) X. Zhang, Z. Chi, H. Li, B. Xu, X. Li, W. Zhou, S. Liu, Y. Zhang and J. Xu, Chem. - Asian J., 2011, 6, 808.

21 Z. Zhao, H. Nie, C. Ge, Y. Cai, Y. Xiong, J. Qi, W. Wu, R. T. K. Kwok, X. Gao, A. Qin, J. W. Y. Lam and B. Z. Tang, Adv. Sci., 2017, 4, 1700005. 